Pragmatism and operationalism

\title{
As pragmatic as theft over honest toil: Disentangling pragmatism from operationalism
}

\author{
Andrew Maul \\ University of California, Santa Barbara \\ Joshua McGrane
}

Oxford University Centre for Educational Assessment (OUCEA), Department of Education, University of Oxford

Correspondence concerning this article should be addressed to Andrew Maul, Gevirtz Graduate School of Education, University of California, Santa Barbara. E-mail: amaul@ucsb.edu. 
"The uniformity of weights and measures cannot displease anyone but those lawyers who fear a diminution in the number of trials, and those merchants who fear anything that renders the operations of commerce easy and simple" (Condorcet, 1793, cited in Hand, 2016, p.6)

The concept of pragmatism has come to garner widespread appeal over the past century, particularly in the context of methodological innovations in the social and psychological sciences. In addition to connoting an orientation towards practicality and realworld usefulness - and who among us would not want to claim such things - pragmatism is often used to suggest freedom from slavish devotion to abstract principles or theoretical concerns. The assumption of this freedom has led to a diversification of activities being referred to as measurement across different scientific disciplines and other areas of human activity. Many of these activities bear little resemblance to canonical instances of measurement in the physical sciences (e.g., the measurement of length, or temperature). Pragmatism, then, may seem to offer an alternative method of justification for the dependability and usefulness of the knowledge acquired as a result of these more liberallydefined measurement activities.

Of course, whether pragmatism actually succeeds in offering such a justification is another matter. As a philosophical approach to truth and knowledge, the Pragmatist school may have much to offer to measurement theorists. However, in many contexts the idea of pragmatism is invoked but not carefully defined. One obvious danger resulting from this is that different users of the term will have different intuitions about what it means, raising the possibility that the term is used in a manner that is explanatorily soothing, but semantically and scientifically vacuous. This vacuum may be easily filled by anti-realist positions that ultimately serve no pragmatic purpose other than to render the operations of measurement deceptively easy and simplistic. 


\section{"Pragmatic" and "representational" approaches to measurement}

In a recent volume entitled Measurement: A Very Short Introduction, David Hand (2016) provides a remarkably compact survey of measurement theory and practice across many areas of scholarship and application, including physical, biological, psychological and social systems. The sheer breadth of the discussion of different measurement applications is a major strength of the volume, and one not commonly encountered in introductory texts on measurement. This affords the reader the opportunity to reflect on how the logic and practice of measurement has been taken up in each of these areas, and in so doing, also provides an opportunity to notice potential incoherencies.

Hand proposes that approaches to measurement across these different areas of inquiry can be located along a continuum from "representational" to "pragmatic." Across the two introductory chapters, Hand offers some conceptual explanation of the distinction between the representational and pragmatic approaches in terms of whether one is attempting to model empirical objects and relationships by mapping them to a numerical representation, or attempting to construct attributes of interest by some procedure with properties that are appropriate for some practical use or purpose. Aside from this, Hand gives a mostly ostensive definition of this continuum, referring to classical instances of measurement in the physical sciences (e.g., the measurement of length) as examples of measurement on the representational end, whereas procedures for efficiently, numerically summarizing large amounts of situationally-relevant information (e.g., the Apgar score used to assess the health of infants) are located on the pragmatic end.

While this simple summation of different purported measurement approaches holds intuitive appeal, a closer inspection suggests that its usefulness is limited by a lack of definitional clarity — and as a result, the continuum may serve more as a post-factum pardon of some conceptual errors that are commonly observed in examples of "pragmatic 
measurement." This lack of clarity is particularly reflected in the ambiguous use of the word "pragmatic" throughout the volume: at times it is associated or identified with subjectivity (e.g., p.76), conventionalism (e.g., p.17), constructivism (e.g., p.17, 34), arbitrariness (e.g., p.4, 53), norm-referencing (p.70), non-realism (p.28), non-physical realism (p.65), a lack of concern with understanding (p.75), and summarization or data reduction (e.g., p.66, 88). Most of all, though, pragmatism seems to be used as a stand-in for a particular interpretation of operationalism, or the semantic doctrine that "the concept is synonymous with the corresponding set of operations" (Bridgman, 1927, cited in Hand, p.27). This particular reading of operationalism, which in our experience is commonly invoked throughout the social and psychological sciences (especially in informal settings), invokes a form of antirealism. This is reflected in comments such as "pragmatic measurement simultaneously defines the attribute being measured and specifies how to measure it” (Hand, p.27), and, regarding a measure of well-being, "precisely what they [individual questions] are measuring is defined solely by the questions themselves...In contrast to the representational case there is no more fundamental 'reality' which can be appealed to" (p.28). Thus, the test constructor appears to be excused from needing to specify what a test measures or how it operates; as long as one has a (number-generating) set of operations, measurement simply takes place by fiat.

Hand (p.30) attempts to qualify this operationalist underpinning to "pragmatic measurement" when he states that it "does not mean 'anything goes'.” However, when measurement is freed from any reality constraints, the inevitable outcome is (as repeatedly demonstrated throughout the social and psychological sciences, and Hand's volume) that anything does go. Nonetheless, Hand's apprehension is well founded, as this severe formulation of operationalism has been met with widespread criticism and rejection from measurement theorists, philosophers of science, and working scientists, including Bridgman 
Pragmatism and operationalism

himself ("I feel that I have created a Frankenstein, which has certainly got away from me", as he once lamented; Bridgman in Frank, 1956, p. 75-76). Although a thorough account of this history is not possible here (though see, e.g., Chang, 2009), the central deficiency of operationalism can be noted by observing that attempting to deny that measurement involves a connection to a "more fundamental reality" quickly leads to incoherencies, as reflected, for example, in allusions to operationally-defined measures "capturing different aspects" (Hand, p.111) of a target phenomenon — which must exist independently of any measurement procedure for such a phrase to be considered coherent. Although Hand is certainly correct that there may be a fair amount of user's choice involved in the construction of an assessment procedure (for example, one may choose which criteria to include in an index of health such as an Apgar score, or how to weigh different economic indicators in an index such as the Dow Jones Industrial Average), the usefulness of such procedures is ultimately underwritten by the fact that they convey information about true (albeit complex) states of affairs (e.g., about an infant or an economy).

In summary, even the most "pragmatic" instances of measurement aim to represent reality, and thus require an empirical connection to reality. Combined with Hand's (p.17) converse admission that even instances of measurement at the extreme "representational" end of the continuum involve pragmatic elements, one seems to be left with the conclusion that this continuum is false. Moreover, as will be argued in the following section, it seems to miss the point of pragmatism itself.

\section{What can pragmatism offer measurement?}

Pragmatism was and is a philosophical movement concerned with truth and epistemology, focused around the idea that the purpose of beliefs is to facilitate successful engagement with the world. Originally formulated by scholars such as Peirce, James, and 
Dewey, the pragmatic maxim was "to try to interpret each notion by tracing its respective practical consequences" (James, 1907, p.28). As such, the pragmatic orientation asks an investigator to see which ideas are useful - that is, to empirically investigate the consequences of holding one belief over another.

Sherry (2011) provides an example of how the pragmatic maxim can be invoked in the context of measurement. He argues that a belief about measurement - in particular, the belief that differences in levels of a property (e.g., temperature or intelligence) are quantitative and therefore measurable in the classical sense of the term - can be evaluated pragmatically by investigating the usefulness of one's endorsement of it, over a weaker hypothesis, such as that that differences in levels of a property are only ordinal. Sherry exemplifies this with reference to the historical progression of measurement of temperature, noting that the transition in the 18th century from the modeling of temperature as ordinal to its modeling as quantitative was justified by showing that there were useful consequences of the latter perspective: specifically, that it led to the development of "new thermal concepts [specific heat and latent heat] useful for explaining and predicting thermal phenomena" (p.516), which in turn led to advances in theory about the natural world. Additionally, these advances offered a new perspective on the problem of coordination of multiple instruments sensitive to differences in temperature (e.g., calibrated glass tubes filled with mercury versus alcohol): since different types of fluid expand at different (and non-linearly-related) rates when heated, it was previously claimed by some that there could be no non-arbitrary method for determining equality among temperature intervals (e.g., Mach, 1896, discussed in Tal, 2013) - from which Hand (p.53) concludes that "temperature scales defined in such ways are heavily pragmatic," invoking the identification of pragmatism with operationalism (p.27). But, ironically, it was the pragmatic demonstration that a specific strategy for modeling temperature as quantitative had tangible benefits that provided an external justification for 
this choice, bringing unification and coherence to thermodynamic theory. In other words, the application of a pragmatic orientation towards measurement prompted a move away from arbitrary numerical assignments, away from operationalism, and towards a more accurate representation of reality.

Pragmatism, as a philosophical movement, encourages an emphasis on the demonstration of the usefulness of ideas - as Sherry (2011, p.523) notes, "acceptance of the pragmatic approach to quantification does not, of course, entail the existence of quantitative psychological attributes... the pragmatic value must be demonstrated.” This stands in stark contrast to the empty way in which pragmatism is often discussed in the contexts of social, economic and psychological measurement, which Hand's otherwise impressive volume unfortunately propagates. Conversely, at several points in the volume (e.g., p.74), Hand notes that building accurate and useful measures in any scientific discipline is an enormous undertaking, likely requiring years of dedicated work. Pragmatism can play a role in such undertakings by offering a perspective on where and how to look, but not on what to see. For that, there may ultimately be nothing more pragmatic than honest scientific toil. 
Pragmatism and operationalism

\section{References}

Chang, Hasok, "Operationalism", The Stanford Encyclopedia of Philosophy (Fall 2009 Edition), Edward N. Zalta (ed.), URL = <http://plato.stanford.edu/archives/fall2009/entries/operationalism/>.

Frank, P. (1956). The Validation of Scientific Theories. Boston: Beacon Press.

Hand, D. (2016). Measurement: A Very Short Introduction. Oxford University Press.

James, W. (1907). Pragmatism: A new name for some old ways of thinking. Cambridge MA: Harvard University Press.

Sherry, D. (2011). Thermoscopes, thermometers, and the foundations of measurement. Studies in the History and Philosophy of Science, 42, 509-524.

Tal, E. (2013). Old and new problems in the philosophy of measurement. Philosophy Compass, 8, 1159-1173. 